\title{
Taguchi Optimization of Plasticisation Process for Glycerol and TEA Based Thermoplastic Mango Starch Biofilms
}

Ernest Chiedoziam Agwamba ( $\sim$ agwamba.ernest@gmail.com )

Clifford University Owerrinta Abia NG https://orcid.org/0000-0002-8008-9572

Lawal G. Hassan

Usmanu Danfodiyo University Sokoto

Achor Muhammad

Usmanu Danfodiyo University Sokoto

Abdullahi M. Sokoto

Usmanu Danfodiyo University Sokoto

Eric N. Agwamba

Kebbi State University of Science and Technology

\section{Research Article}

Keywords: Plasticization, Taguchi Technique, young modulus, Main effect plot, Signal-to-noise ratio, Interaction plot

Posted Date: September 28th, 2021

DOI: https://doi.org/10.21203/rs.3.rs-932373/v1

License: (c) (1) This work is licensed under a Creative Commons Attribution 4.0 International License. Read Full License 


\section{Abstract}

This investigation involves study of independent variable that influences the Young modulus of thermoplastic mango starch (TPS) as dependent response factor. The experiment was design using the Taguchi orthogonal technique with four independent variables; plasticiser type; glycerol (G), and Triethanolamine-(TEA) $(T)$, percentage plasticiser (40 and $120 \%$ ), percentage carboxymethyl celluloseCMC (10 and $50 \%)$, and concentration of $\mathrm{HCl}(0.05$ and $0.15 \mathrm{M})$. The result of the main effect plots for mean indicated that the gTPS-CMC1 with $268.85^{\mathrm{a}} \mathrm{MPa}$ is a better outcome compared to gTPS-CMC3 with $280.31^{\mathrm{a}} \mathrm{MPa}$, since no significance difference was observed due to less composition requirement of $\mathrm{CMC}$ for gTPS-CMC1, making it more cost effective to produced with better optimum conditions. The interaction plot of the independent variables showed that for plasticiser types; when glycerol (G) was utilised a higher young modulus is observed than TEA $(T)$ and only interacts with TEA (T) at $0.015 \mathrm{M} \mathrm{HCl}$; $10 \% \mathrm{CMC}$ gives a higher response compared to $50 \% \mathrm{CMC}$ and showed no interaction even as the other independent variables fluctuates, and similar effect was observed for percentage plasticiser. Study concluded that the predicted mean (young modulus) is substantially consistent with the experimental observation $\left(R^{2}=0.6283\right)$.

\section{Introduction}

The level of pollution cause by petro-plastics and high costs of production associated with bioplastic has been a disadvantage that has driven research to look for more environmental and economical alternatives. Most research is carried out without the application of statistical procedure that could help in estimating and eliminating trial and error, minimizing wastage of resources and time in production procedures ${ }^{[1,2]}$. Challenge facing the up-scaling commercial production of bioplastic could be due to lack of comprehensive feasibility report on the full market potential, and high cost associated with certain class of bioplastics ${ }^{[3,4]}$. The over dependence on unsustainable resources ${ }^{[5]}$ and time consumption could be attributed to poor experimental approach, technique, design and insufficient information on biomaterials-biopolymers. Environmental concerns has gradually wiped up sentiments, with public perception and awareness on the rise, and government policy aligning towards promoting sustainable approaches that are environmental friendly, economical, and efficient ${ }^{[6]}$. The utilization of bioplastics is a step forward innovation to solving some of the environmental issues using cost-effective renewable substrates ${ }^{[7]}$. This has prompted studies into the possibility of deriving raw materials from abundance agricultural by-product as feedstock for large-scale bioplastic production ${ }^{[5]}$ with low-cost ${ }^{[8]}$, and excellent performance ${ }^{[9]}$. In addition, the production cost could be mitigated when the proper scientific approached is employed by using appropriate experiment design before commercial scale up.

With all the above factors in perspective the most viable bioplastic with potential for commercialisation are thermoplastic derived from biomass through thermomechanical process known as plasticisation. Plasticisation process can be designed with robust mathematical model that produces optimum condition for cost effective thermoplastic biomass production. Plasticisation of biomass to bioplastic 
remains one the affordable route to obtaining sustainable plastics due to its simplicity and versatility [10, 11]. As it allowed the use of variety of plasticiser(s) to convert rigid biomass into flexible bioplastic refer to as thermoplastic ${ }^{[12]}$. The process does not release any harmful by-product(s) into the environment and gives a material that is renewable ${ }^{[13]}$. Enhanced, economical, and efficient plasticisation process can help in producing thermoplastic starch with commercial value when a design of experiments (DOE) model is employed to derive the optimum conditions and predicts experimental outcome ${ }^{[14]}$. Scaling up the process and procedure for the production could help in creating a profitable commercial venture of thermoplastic starch if the appropriate approach of laboratory experimental design is employed which does not depend on single independent variable as practiced ${ }^{[15]}$.

Therefore, the use of DOE represents a better alternative approach in scientific research compared to traditional or conventional technique that only put experimental outputs consideration. Most times the ttest, chi-test, analysis of variance (ANOVA) are applied to study outcomes of research investigations to ascertain certain relevant information that could be used for up-scale process and production in commercial ventures ${ }^{[16]}$. These provided information about significant difference in research data which is not enough most times, provides no information about economical advantage since it does not consider the independent input production condition(s) and product composition(s) in analysing the defined response dependent variable which is just an output.

The common types of DOE methods have their advantages over one another and there includes Response surface, Factorial, Mixture, and Taguchi methods [17]. These techniques could predict the input conditions and there corresponding output responses with response optimizer but will not give the optimum output experimental response except for Taguchi methods that does not predicts input conditions but gives the optimum experimental output response and predicts outcome response based on the independent variables ${ }^{[16]}$. This technique measures the robustness utilised to recognize control factors and reduces unpredictability in product(s) or process(s) by minimizing the effects of uncontrollable factors (noise factors) ${ }^{[15,17]}$.

Therefore, the Taguchi technique is a simple but robust method that provides direct optimum conditions for profitable production ${ }^{[15]}$. Taguchi provides information on optimum conditions that is usually profit oriented when applied either in small, medium or large scale production due to ability to selected conditions that are cost effective in thermoplastic starch production, and can help estimate both the absolute and relative error by providing predicted outcomes that can be comparable with experimental output ${ }^{[14,15]}$. Vital information that could be derived from Taguchi technique includes; Main effect plot for Signal/Ratio and Mean, Interaction plot, predicted mean (PMEAN). Therefore, the aim of this investigation is to design experimental procedure and ascertain the optimum condition for the plasticization of Mango starch with glycerol and TEA, with young modulus as the dependent variable response applying larger-is-better Taguchi technique equation model.

\section{Materials And Methods}




\subsection{Materials}

The mango starch was provided by the Department of Pharmaceutics, Faculty of Pharmaceutical Science, Usmanu Danfodiyo University Sokoto, Sokoto, Nigeria and other reagents and tensionmeter was provided by Department of Chemical Sciences, Faculty of Sciences, Clifford University, Owerrinta, Abia State, Nigeria. All chemicals and reagents were used without any further purification.

\subsection{Methods}

\subsubsection{Taguchi Design of Experiment}

Taguchi Orthogonal Array Design, L8(2^4), level : 2, Factors: 4, Runs: 8, and Columns of L8(2^7) Array 1 , $2,4,5$. The Design contains 3 numerical factors (controllable), and one categorical factor plasticiser type (uncontrollable factor) as shown in Table 2.1.

Table 2.1

Taguchi Design indicating the Composition of thermoplastic mango starch biofilms

\begin{tabular}{|lllll|}
\hline Sample Code & Plasticiser type & \%CMC & \%Plasticiser & HCl (M) \\
\hline gTPS-CMC1 & G & 10 & 40 & 0.05 \\
\hline gTPS-CMC2 & G & 10 & 120 & 0.15 \\
\hline gTPS-CMC3 & G & 50 & 40 & 0.05 \\
\hline gTPS-CMC4 & G & 50 & 120 & 0.15 \\
\hline tTPS-CMC1 & T & 10 & 40 & 0.15 \\
\hline tTPS-CMC2 & $T$ & 10 & 120 & 0.05 \\
\hline tTPS-CMC3 & $T$ & 50 & 40 & 0.15 \\
\hline tTPS-CMC4 & T & 50 & 120 & 0.05 \\
\hline $\begin{array}{l}\text { gTPS-CMC: } \text { glycerol plasticised thermoplastic mango starch reinforced with Carboxymethyl cellulose, } \\
\text { tTPS-CMC: TEA plasticised thermoplastic mango starch-reinforced with Carboxymethyl cellulose, G: } \\
\text { glycerol, T: TEA }\end{array}$ & & & \\
\hline
\end{tabular}

\section{2.2. Preparation Of Thermoplastic Mango Starch Biofilm}

The thermoplastic starch biofilm was prepared based on method described in previous publication [18] and casting technique was use to make the final biofilms by pouring the hot liquid plasticised thermoplastic starch mixture into pre-labelled Petri-dish and allowed to stand on laboratory bench for 48 hours. The composition of each thermoplastic mango starch biofilms are shown in Table 1 as derived from the Taguchi Orthogonal array design. 


\section{2.3. Measurement of Young modulus}

The TPS-CMC biofilms were cut into a dumb-bell shape with the help of a metallic template and the length $\left(I_{1}\right)$ and breadth $(b)$ measure with the help of a metre-ruler. The dumb-bell shape biofilms was clamped firmly unto a fabricated tensionmeter at both ends. The weight (w) needed to break the TPSCMC biofilms at the middle and new length $\left(\mathrm{I}_{2}\right)$ before breaking was measured and recorded in triplicates ${ }^{[19]}$.

Stress $=$ Force $(\mathrm{N}) /$ Area $\left(\mathrm{m}^{2}\right)$

Where Force $=\mathrm{w} \times 9.86$, and Area $=I_{1} \times b$

Convert Stress from $\mathrm{Pa}$ to $\mathrm{MPa} .\left(\mathrm{Pa}=1 \times 10^{-6} \mathrm{MPa}\right)$

Strain $=\underline{1_{2}(\mathrm{~m})-1_{1}(\mathrm{~m})}$ $1_{1}(\mathrm{~m})$

Young modulus $=\underline{\text { Stress }(\mathrm{MPa})}$

Strain

\section{2.4. Measurement of Absolute and Relative Error}

Mathematical the absolute and relative error are determined using equation shown below [20, 21].

$A \cdot E=P M E A N-E M E A N$

$\%$ R.E. $=\frac{\text { A.E }}{\text { PMEAN }} \times 100$

Where A.E: absolute error, R.E: Relative error, PMEAN: Predicted Mean (Young modulus), and EMEAN: Experimental Mean (young modulus).

\section{2.5. Statistical Analysis}

The observed experimental outcome (YM) was analysed using Analysis of variance (ANOVA) to determine the significant difference at $95 \%$ confidence interval. The Taguchi designed experiment was optimized using two step optimization processes. Step 1 used the Larger-is-better signal-to-noise ratio $(\mathrm{S} / \mathrm{N})$ to identify those factors that reduces variability to maximise the response calculated as shown in equation (6). Steps 2 recognized control variables which shift the Mean to objective and have a small or no effect on the signal-to-noise ratio ${ }^{[17]}$. The main effect plot of mean and Signal-noise ratio, interaction plot, and the predicted mean i.e. predicted young modulus where derived and analysed using the higheris better signal-to-noise ratio model of Taguchi method in Minitab 17 software suite.

$\mathrm{S} / \mathrm{N}:-10 \times \log \left[\sum\left(1 / \mathrm{Y}^{2}\right) / \mathrm{n}\right]$ 
Where $\mathrm{Y}=$ response for a given factor level, $\mathrm{n}=$ sum of responses in the factor level combination.

\section{Result}

Table 3.1 indicates the result of Young modulus derived from the experimental determination while Table 3.2 and 3.3 shows the Taguchi Analysis of Young modulus response with respect to the independent variable Plasticiser type, $\mathrm{CMC}(\%)$, Plasticiser (\%), and $\mathrm{HCl}(\mathrm{M})$.

Table 3.1

Young Modulus (Response) with Grouping Information using the Tukey Method at 95\% Confidence Interval

\begin{tabular}{|c|c|c|c|c|c|}
\hline Sample Code & Plasticiser type & $\mathrm{CMC}(\%)$ & Plasticiser (\%) & $\begin{array}{l}\mathrm{HCl} \\
\text { (M) }\end{array}$ & $\begin{array}{l}\text { Response } \\
\text { (Young Modulus) } \\
\text { (MPa) }\end{array}$ \\
\hline gTPS-CMC1 & G & 10 & 40 & 0.05 & $268.849 \pm 01.569^{a}$ \\
\hline gTPS-CMC2 & G & 10 & 120 & 0.15 & $155.596 \pm 27.369^{b c}$ \\
\hline gTPS-CMC3 & G & 50 & 40 & 0.05 & $280.309 \pm 08.280^{a}$ \\
\hline gTPS-CMC4 & G & 50 & 120 & 0.15 & $091.114 \pm 01.791^{\mathrm{de}}$ \\
\hline tTPS-CMC1 & $\mathrm{T}$ & 10 & 40 & 0.15 & $176.404 \pm 13.785^{b}$ \\
\hline tTPS-CMC2 & $\mathrm{T}$ & 10 & 120 & 0.05 & $124.993 \pm 39.413^{\mathrm{cd}}$ \\
\hline tTPS-CMC3 & $\mathrm{T}$ & 50 & 40 & 0.15 & $106.636 \pm 02.734^{\mathrm{cd}}$ \\
\hline tTPS-CMC4 & $\mathrm{T}$ & 50 & 120 & 0.05 & $041.347 \pm 05.161^{e}$ \\
\hline
\end{tabular}

Table 3.2

Response Table for Larger-is Better Signal-to-Noise Ratios

\begin{tabular}{|lllll|}
\hline Level & $\begin{array}{l}\text { Plasticiser } \\
\text { type }\end{array}$ & \% $\mathrm{MC}$ & \% Plasticiser & $\mathrm{HCl}(\mathrm{M})$ \\
\hline 1 & 45.14 & 44.82 & 45.76 & 42.95 \\
\hline 2 & 39.94 & 40.26 & 39.32 & 42.13 \\
\hline Delta & 5.20 & 4.57 & 6.43 & 0.82 \\
\hline Rank & 2 & 3 & 1 & 4 \\
\hline
\end{tabular}


Table 3.3

Response Table for Means

\begin{tabular}{|lllll|}
\hline Level & $\begin{array}{l}\text { Plasticiser } \\
\text { Type }\end{array}$ & \% CMC & \% Plasticiser & $\mathrm{HCl}(\mathrm{M})$ \\
\hline 1 & 199.0 & 181.5 & 208.1 & 178.9 \\
2 & 112.3 & 129.9 & 103.3 & 132.4 \\
Delta & 86.6 & 51.6 & 104.8 & 46.4 \\
Rank & 2 & 3 & 1 & 4 \\
\hline
\end{tabular}

\section{Discussion}

From Table 3.1, the result should that gTPS-CMC3 has the highest young modulus of $280.31 \mathrm{MPa}$ (response) compared to gTPS-CMC1 of $268.85 \mathrm{MPa}$ but are not significant difference $(p>0.05)$, but both are significantly higher compared to the biofilm derivatives ( $p \leq 0.05)$. $155.596 \mathrm{MPa}$ was observed for gTPS-CMC2 that is less than $176.40 \mathrm{MPa}$ for tTPS-CMC1, and higher than $124.99 \mathrm{MPa}$ for tTPS-CMC2, and 106.64 MPa for tTPS-CMC3 but indicates no significant differemce $(p>0.05)$, while gTPS-CMC4, tTPS-CMC2 and tTPS-CMC3 showed no significant difference $(p>0.05)$ but are significantly lower than tTPS-CMC1 ( $p \leq 0.05$ ). 0.41.35 MPa was observed for tTPS-CMC4 is significantly the lowest response except for gTPS-CMC4 with $0.91 \mathrm{MPa}$ where is showed not significant difference $(p>0.05)$.

Table 3.2 and 3.3 are consistent with each other from the Delta ranking of the controllable factors on the mean and signal-to-noise ratio, the both table indicates that plasticiser (\%) has the highest effect on the Mean and Signal to Noise ratio. Followed by Plasticiser type, and thirdly $\mathrm{CMC}(\%)$ and lastly with $\mathrm{HCl}$. This observation is expected to be consistent with main plot mean and signal-to-noise ratio.

From Fig. 3.1 and 3.2, the main effect plot of mean and signal-to-noise ration showed that glycerol plasticised thermoplastic mango starch hydrolysed with $0.05 \mathrm{M} \mathrm{HCl}$, and plasticised with $40 \%$ glycerol and reinforced with $10 \% \mathrm{CMC}$ indicates the optimum condition for the production of $\mathrm{CMC}$ reinforced thermoplastic mango starch which is equivalent to gTPS-CMC1 with young modulus i.e. mean of 268.85 MPa and signal-to-noise ratio 51.055. The results indicates that selection of optimun conditions is factor that depends on the smallest input of independent variablethat gives the highest output response. Result indicates that gTPS-CMC1 with $268.85 \mathrm{MPa}$ is lower than gTPS-CMC3 with $280.31 \mathrm{MPa}$ signal-to-noise ratio but the anaysis of variance reveal that both outcomes are thesame but the choice of gTPS-CMC1 byTaguchi approache over gTPS-CMC3 does not not depend on the highest output response but on the lowest possible combination of inputs i.e. independent variables that gives a output that are signifcantly viable or economical to produced. gTPS-CMC3 will required 50\% CMC, $40 \%$ glycerol and $0.05 \mathrm{M} \mathrm{HCl}$ to give $280.31 \mathrm{MPa}$; and gTPS-CMC1: $10 \% \mathrm{CMC}, 40 \%$ glycerol and $0.05 \mathrm{M} \mathrm{HCl}$ is required to give 268.85 MPa. the choise of gTPS-CMC1 over gTPS-CMC3 lies in the amount of CMC reuquired as input from the main effect plot, because the amount of required to give 280.31 MPa gTPS-CMC3 is $50 \%$, while $10 \%$ is 
required to produced 268.85 MPa for gTPS-CMC1 with no significant difference in outcomes $(p>0.05)$. this represent a reduction in production cost since a lesser amount of $\mathrm{CMC}$ is required to produced gTPS$\mathrm{CMC1}$. The observation is consistent with previous study on carboxymethylation process using Taguchi technique by Agwamba et al ${ }^{[22]}$.

Figure 3.3 showed that type $G$ and $T$ has no interaction between then when $C M C$ increases from 10 to 50 $\%$. Increase in plasticiser 40 to $120 \%$, but when the $\mathrm{HCl}$ was increased from, 0.05 to $0.15 \mathrm{M}$, the $\mathrm{G}$ and $\mathrm{T}$ interacted at $0.15 \mathrm{M} \mathrm{HCl}$. The $\mathrm{CMC}$ at 10 and $50 \%$ showed no interaction with $\mathrm{G}$ and $\mathrm{T}, 40$ to $120 \%$ plasticiser, and $0.05 \mathrm{M}$ to $0.15 \mathrm{M} \mathrm{HCl}$. The \% Plasticiser at 40 and $120 \%$ showed no interaction from for plasticised type from $\mathrm{G}$ and $\mathrm{T}, \% \mathrm{CMC}$ from 10 to $50 \%$, and $\mathrm{HCl}$ from 0.05 to $0.15 \mathrm{M}$. $\mathrm{HCl}$ at 0.05 and 0.15 $\mathrm{M}$ showed no interaction for $\mathrm{CMC}$ from 10 to $50 \%$ but showed interacted at $\mathrm{T}$ for plasticised type, and $120 \%$ plasticiser.

According to Henseler et al., ${ }^{[23]}$, the $\mathrm{R}^{2}$ value of 0.6283 observed in this study indicates that the predicted mean is substantially consistent with the measured experimental observation. Three (3) of the observed mean (predicted and experimental) i.e. the predicted mean of gTPS-CMC1, gTPS-CMC3, tTPS-CMC2, and tTPS-CMC4 are significantly different from the experimental mean $(p \leq 0.05)$ which represents about 37.5 $\%$ of the result indicating that the remaining $62.5 \%$ are not significant different $(p>0.05)$. This showed that the model fit for deriving predicted mean.

The Fig. 3.4, also indicated that the predicted mean and experimental mean of gTPS-CMC1, gTPS-CMC3, tTPS-CMC2, and tTPS-CMC4 are significantly different $(p \leq 0.05)$ because the relative error are approximately higher than $10 \%$ and gTPS-CMC2, gTPS-CMC4, tTPS-CMC1, and tTPS-CMC3 indicated a relative error that are approximately less than $10 \%$ and are not significantly different $(p>0.05)$. Therefore, the Taguchi technique model could be modified to help estimate of level accuracy in an experimental results. The relative error derived from the Taguchi Prediction model relates to the significance difference between the predicted and experimental mean derived from the analysis of variance (ANOVA) of experimental mean response.

\section{Conclusion}

The use of Taguchi technique for design and analysis gives the optimum independent production parameters, and predict almost accurately the response. This represents an efficient and economical avenue to mitigate the expensive nature associated with bioplastic processing and production in either large or small scale and if emulated can help reduce cost production in commercial venture. Furthermore, the larger-is-better signal-to-noise model can be modified to include relative error, which could further help give credence to the outcome of scientific investigation. Therefore, it can be said that Taguchi technique is a versatile tool that could be use for optimization and estimate errors in outcome of plasticisation process of glycerol and TEA plasticised thermoplastic mango starch with optimum conditions that could be used for up-scale commercial production. 


\section{Declarations}

Acknowledgments: The authors wish to sincerely thank all who assisted in preparation of this work.

Author Contributions: Authors contributed to all phases of the elaboration of the paper.

Conflicts of Interest: There is no conflict of interest.

Funding: This research did not receive any specific grant from funding agencies in the public, commercial, or not-for - profit sectors.

\section{References}


[1] J. H. Skinner, "Solid Waste: Assessment, Monitoring and Remediation," in Waste Management Series, I. Twardowska, Ed., Elsevier B.V, 2004, pp. 1091-1098.

[2] P. N. Cheremisinoff, "Waste Reduction," in Waste Minimization and Cost Reduction for the Process Industries, William Andrew Applied Science Publisher, 1995, pp. 1-51.

[3] V. Bui, D. Park and Y. Lee, "Chitosan Combined with $\mathrm{ZnO} \mathrm{TiO}_{2}$ and Ag Nanoparticles for Antimicrobial Wound Healing Applications: A Mini Review of the Research Trends.," Polymers, vol. 9, no. 21, pp. 2-25., 2017.

[4] O. Vilpoux and L. Averous, "Chapter 18 Starch-Based Plastics. Didalam: Book 3-Technology, use and potentialities of Latin American Tubers," Copenhagen, 2004.

[5] Science for Environment Policy, "Enhancing Qualities of Starch-based Bioplastic.," European Commission DG Environment News Alert Service. Edited by SCU. The University of New West England,(295):, Bristol, 2012.

[6] United Nations, "Achieving Sustainable Development and Promoting Development Cooperation: Dialogues at the Economic and Social Council," United Nations, New York, 2008.

[7] S. M. Maulida and P. Tarigan, "Production of Starch Based Bioplastic from Cassava Peel Reinforced with Microcrystalline Cellulose Avicel PH101 using Sorbitol as Plasticiser.," Journal of Physics: Conference Series, vol. 710, pp. 1-7, 2016.

[8] R. Jain and A. Tiwari, "Biosynthesis of Planet Friendly Bioplastics using Renewable Carbon Source," Journal of Environmental Health Science, vol. 13, pp. 1-3, 2015.

[9] B. Mose and S. Maranga, "Mose B. R. and Maranga S.M. (2011). A Review on Starch Based Nanocomposites for Bioplastic Materials," Journal Material Science Engineering, vol. 1, pp. 239-245, 2011.

[10] F. C. de Paula, C. B. de Paula and J. Contiero, Prospective Biodegradable Plastics from Biomass Conversion Processes, Biofuels - State of Development, IntechOpen, 2018.

[11] L. Shen, E. Worrell and M. K. Patel, "Present and future development in plastics from biomass," Biofuels Bioproducts and Biorefining, vol. 4, no. 1, pp. 25-40, 2010.

[12] M. G. A. Vieira, M. A. Silva, L. O. Santos and M. M. Beppu, "Natural-based plasticizers and biopolymer films: A review," European Polymer Journal, vol. 47, no. 3, pp. 254-263, 2011.

[13] The Power of Renewables, "Opportunities and Challenges for China and the United States," The National Academies Press, Washington, DC, 2010.

[14] S. K. Karna, V. Ran, R. V. Singh and R. Sahai, "Application of Taguchi Method in Indian Industry," International Journal of Emerging Technology and Advanced Engineering, vol. 2, no. 11, pp. 387-391, 2012.

[15] A. Azadeh, S. S. Miri-Nargesi, S. M. Goldansaz and N. Zoraghi, "Design and implementation of an integrated Taguchi method for continuous assessment and improvement of Manufacturing Systems," International Journal Advanced Manufacturing Technology, vol. 59:, p. 1073-1089, 2012.

[16] Shmula, "Shmula.com," Shmula LLC, 5 June 2018. [Online]. Available: https://www.shmula.com/taguchi-methods-and-how-they-apply-to-manufacturing/25900/. [Accessed 12 April 2020].

[17] Minitab 18, "Minitab 18 Support," Minitab LLC, 2019. [Online]. Available: support.minitab.com/en-us/mintab/18/help-and-how-to/modeling-statistics/doe/support- 
topics/taguchi-designs/what-is-the-signal-noise-ratio/. [Accessed 10 April 2020].

[18] E. C. Agwamba, L. G. Hassan, A. M. Sokoto and M. Achor, "Investigation Of Mechanical Properties Of Mango Starch Biocomposite Derivatives," Journal Chemical Society of Nigeria, vol. 44, no. 7, pp. $1305-1312,2019$.

[19] J. Wissinger, R. Harris, A. Johnson, C. Ahrenstorff and L. Seifert, "Make it and Break it: Bioplastic from Plant Starch.," University of Minnesota Centre for Sustainable Polymers. A NSF Centre for Chemical Innovation, pp. 1-9, 2018.

[20] M. Hazewinkel, "Theory of Error," in Encyclopedia of Mathematics, S. S. a. B. Media, Ed., Kluver Academic Publishers, 2001.

[21] R. G. Steel and J. H. Torrie, Principle and Procedures of Statistics, with Special Reference to Biological Sciences, McGraw-Hill, 1960.

[22] E. C. Agwamba, L. G. Hassan, M. Achor and A. M. Sokoto, "Taguchi Optimization of Carboxymethylation Process and Effect Reaction Efficiency on Swelling Capacity," Asian Journal of Applied Sciences, vol. 7, no. 5, pp. 528-536, 2019.

[23] J. Henseler, C. Ringle and R. Sinkovics, "The Use of partial least squares path modelling in international marketing," Advances in International Marketing, vol. 20, pp. 277 -320, 2009.

[24] M. Shabina, M. Afzal and S. Hameed, "Bacterial Polyhydroxyalkanoate-Eco-Friendly Next Generation Plastic: Production, Biocompatibility, Biodegradation, Physical Properties and Applications," Green Chemistry Letters and Reviews, vol. 8, no. 3-4, pp. 56-77, 2015.

\section{Figures}

\section{Main Effects Plot for Means (YM) Data Means}

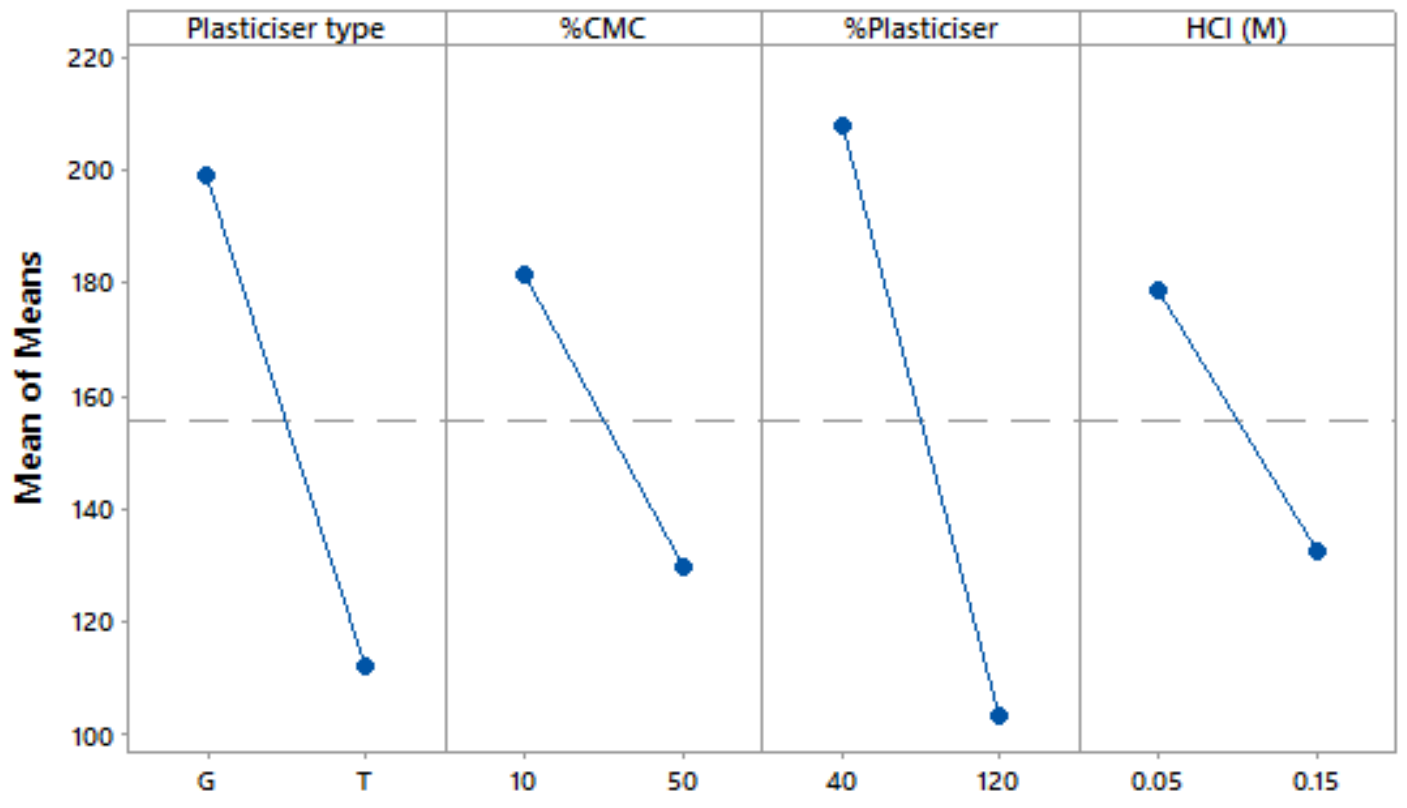

Figure 1 
Main Effects Plot for Mean (Young modulus)

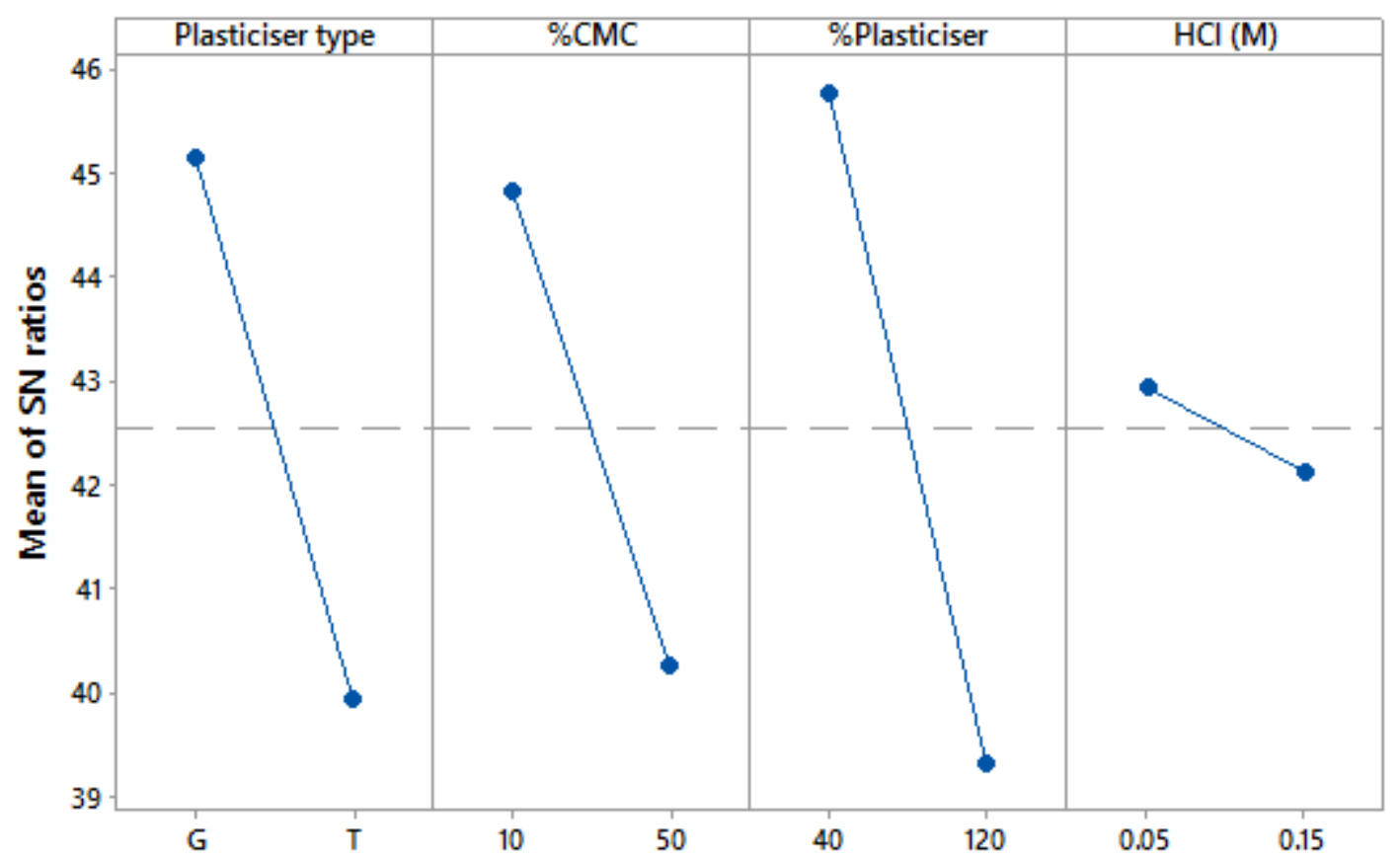

Signal-to-noise: Larger is better

\section{Figure 2}

Data Means Main Effects Plot for Signal-Noise Ratio

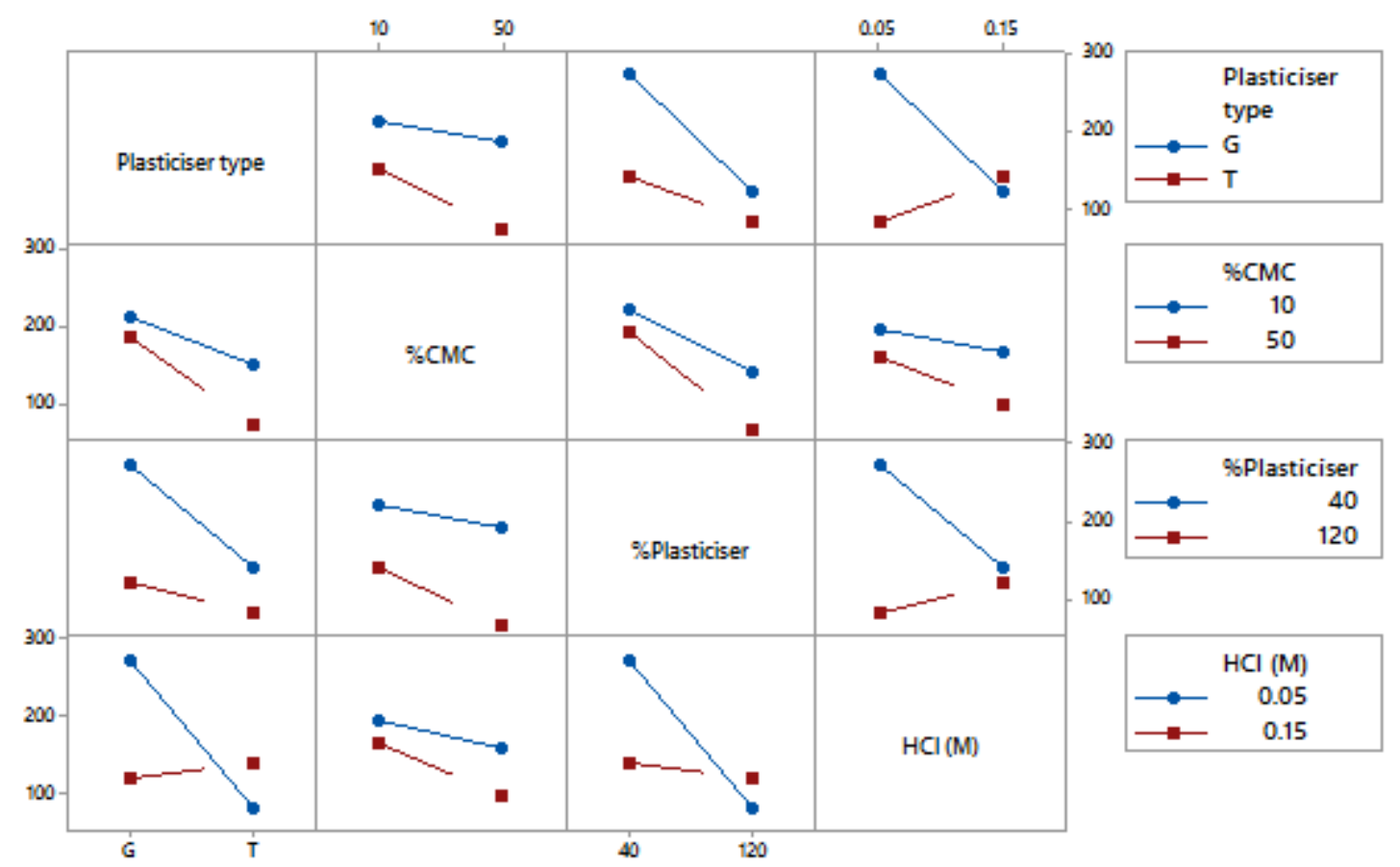

Figure 3 
Data Means Interaction Plot for Young Modulus

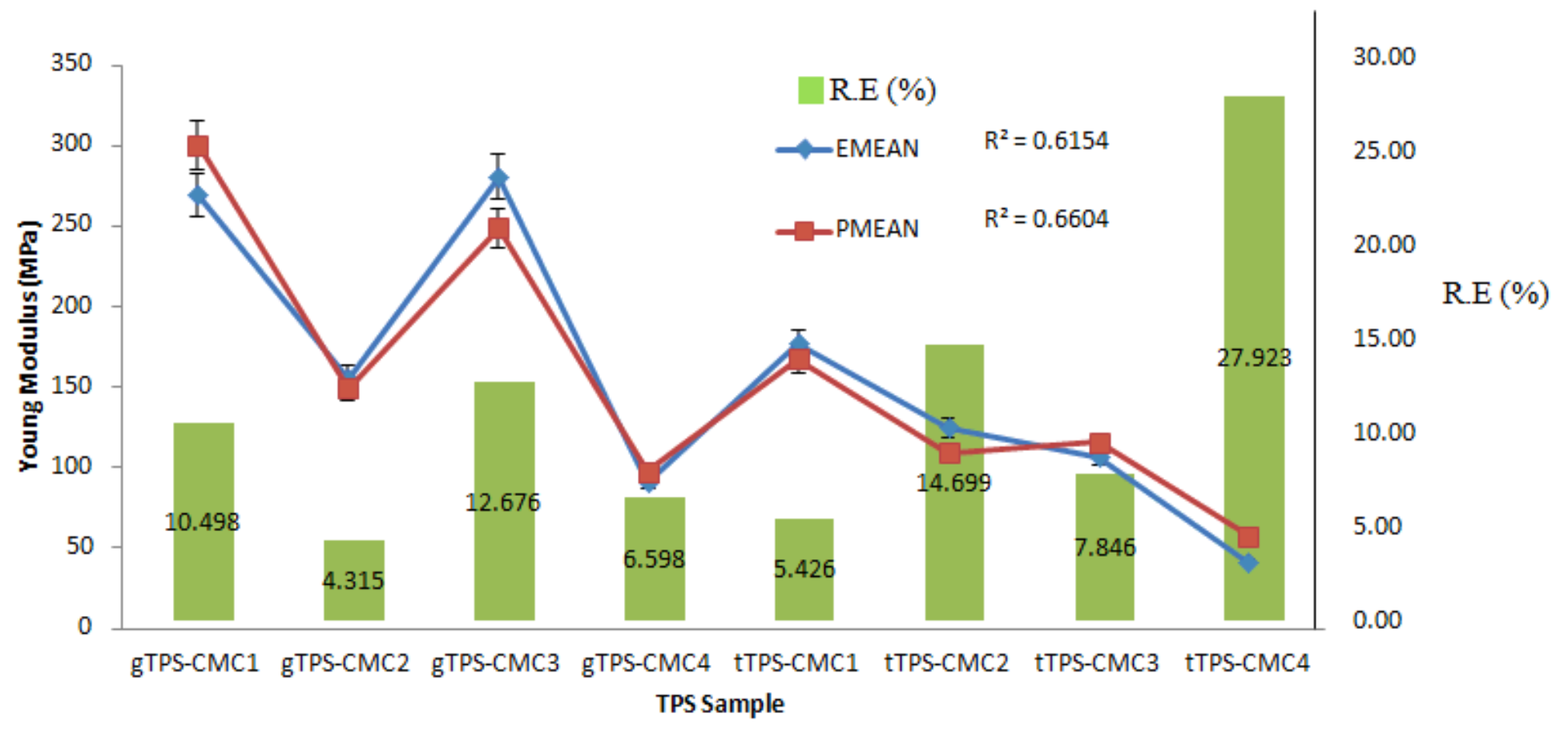

\section{Figure 4}

Experimental and Predicted mean (Young Modulus) and Relative error. Key: R.E is absolute value where R.E: Relative error, PMEAN: Predicted mean and EMEAN: Experimental derived mean. 